\title{
MEASUREMENTS OF THE PERTURBED-INTERPLANETARY \\ MAGNETIC FIELD IN THE LUNAR WAKE \\ by
}

Harold E. Taylor*, K. W. Behannon and N. F. Ness

\author{
NASA-Goddard Space Flight Center \\ Greenbelt, Maryland
}

*NAS-NASA Postdoctoral Resident Research Associate

Extraterrestrial Physics Branch Preprint Series 


\section{ABSTRACT}

Measurements of the interplanetary magnetic field in the vicinity of the moon have been made from lunar orbit on the Explorer 35 spacecraft. They show no shock waves in the vicinity of the moon, either in front of the moon or within $5.4 \mathrm{R}_{M}$ behind the moon. The interplanetary magnetic field appears to be convected past the lunar body without much distortion. A regular pattern of magnetic perturbations is observed when passing through the lunar wake. The perturbation amplitude is small and variable, typically less than $30 \%$ of the ambient interplanetary magnetic field.

Simultaneous measurements made by Explorer 33 while in the interplanetary medium but not in the lunar wake show that even sharp changes in the interplanetary magnetic field are found essentially undistorted in the lunar wake. These simultaneous measurements also help to identify the magnetic pertursation pattern in the wake region. 


\section{INTRODUCTION}

In previous publications (Ness et al., 1967; Ness et al., 1968, hereafter called papers I and II), early results of the GSFC magnetic field experiment on lunar Explorer 35 were reported. The data presented were from the first month after launch and thus covered only a portion of the region around the moon eventually mapped out by Explorer 35. The purpose of this paper is twofold:

1. to present data taken throughout the first six months of spacecraft operation

2. to present simultaneous data from a magnetic field experiment on another spacecraft located in the interplanetary medium in cis-lunar space.

The results of earlier publications (papers $I$ and II, Lyon et al., 1967 and Colburn et al., 1967) are substantiated in this study and will only be briefly treated. Two new observations are reported here.

1. The data show no evidence of a tail shock in the lunar wake (suggested by Michel, 1967) out to aposelene of Explorer 35 , or $5.4 R_{M}\left(R_{M}=1738 \mathrm{~km}\right)$.

2. Simultaneous measurements of the interplanetary magnetic field indicate that inhomogeneities in the interplanetary magnetic field are found essentially undistorted in the plasma wake of the moon. The simultaneous measurements 
are also used to show that the characteristic pattern of magnetic perturbations observed in the lunar wake is due to the presence of the moon, i.e., does not exist in the undisturbed interplanetary medium.

The details of the spacecraft orbit have been given in the earlier reports and will not be repeated here. Representative orbits from the first six months after launch are shown in Figure 1. It can be seen that during this period the spacecraft makes many passes through the lunar wake region from selenocentric radial distances of less than $2 R_{\mathbb{M}}$ to distances greater than $5 \mathrm{R}_{\mathrm{M}}$. All the data presented in this report are taken from periods when the moon and the spacecraft were in the interplanetary medium on the sunward side of the earth's bow shock.

The GSFC magnetic field experiment was described in detail in papers I and II. The data presented here are sequence averages ( 81.8 second averages) of nominally 16 individual vector measurements. The coordinates used are selenocentric solar ecliptic. A description of the data analysis is contained in paper II.

The simultaneous data presented here are from the Explorer 33 spacecraft whose orbit and experiment were described in detail by Behannon (1968). Figure 2 shows the orbit of Explorer 33 as well as the position of the moon during the first lunar month after the launch of Explorer 35. 


\section{DISCUSSION OF THE EXPERIMENTAL OBSERVATIONS}

Examples of the simultaneous measurement of the interplanetary magnetic field are shown in Figure 3 . The solid lines are for Explorer 35 passing through the lunar wake while the dotted lines are for Explorer 33. At 2200 UT on September 9 the two spacecraft were separated by a distance of $29.6 R_{E}$ or $108.5 \mathrm{R}_{M}\left(\mathrm{R}_{\mathrm{E}}=6378 \mathrm{~km}\right)$. From the orbit plot (Figure 2) it is evident that this distance only changes slowly during the time for which data are shown. At 1100 on September 10 the distance had decreased to $25.0 \mathrm{R}_{\mathrm{E}}$ or $91.8 \mathrm{R}_{\mathrm{M}}$.

The magnetic field measurements of the two independent spacecraft are clearly quite similar. In the lunar wake the characteristic pattern of change in the field magnitude is readily identifiable. The magnitude of the field at Explorer 35 is stronger in the plasma umbra and on the outer edges of the penumbra than the corresponding field in the undisturbed solar wind at Explorer 33. Similarly the field is weaker in the penumbral region behind the moon than in the undisturbed interplanetary medium. (The +-+-+ pattern and the concepts of the plasma umbra and penumbra were discussed in paper II.)

The differences in the field at the two spacecraft, while perhaps not as striking as the similarities, are at times larger than the expected experimental errors. This indicates that the differences máy be real, reflecting temporal or spatial structure 
in the magnetic field. Theories of the interaction of the solar wind with the moon (e.g., Whang 1968; Spreiter et al., 1968) predict relatively small directional changes in the field (typically $15^{\circ}$ or less for representative solar wind conditions). Thus it is convenient to regard these differences in direction measured by the two spacecraft as being primarily due to spatial and/or temporal structure in the interplanetary magnetic field and not associated with the lunar wake. (Such an approach is also suggested experimentally by the smallness of the perturbations in the magnetic field magnitude associated with the lunar wake.)

Data from the next two passes of Explorer 35 through the lunar wake are shown in Figure 4, together with the corresponding Explorer 33 measurements. Beside showing the characteristic magnitude perturbations these passes illustrate one of the problems associated with presenting simultaneous data, i.e., the problem of determining appropriate time offsets. In Figure $4 a$ the time offset was chosen to make the temporary increase in the azimuth angle, $\theta$, seen at both spacecraft coincide on the plot. There are few other distinctive features by which this time offset can be checked. This alignment, however, leads to an unusually large time offset. In this case the uncertainty in the time offset does not significantly effect the identification of the magnitude perturbations because the field magnitude at Explorer 33 is essentially constant throughout the 
entire period.

In figure $4 \mathrm{~b}$, some 11.5 hours later, a very small time offset seems to give reasonable correspondence between the two sets of data. It perhaps should be noted that, as indicated in Figure 2, the spacecraft are separated primarily in the solar ecliptic y direction during this period. Thus even if the field is rigidly frozen into the plasma, the existence of spatial structure on a scale length of the order of the satellite separation or less will lead to differences at the two spacecraft. Such spatial structure can also lead to different time offsets at different times. When the spacecraft separation is primarily in solar ecliptic $X$, as occurred late on September 12, 1967, the plasma sampled by one spacecraft is subsequently seen by the other and if the field is truly frozen in identical fields should be seen with a predictable time delay.

Figure 5 a shows a pass of Explorer 35 behind the moon and the corresponding Explorer 33 data on September 12, 1967. At $2000 \mathrm{UT}$ the spacecraft were separated by only $13.0 \mathrm{R}_{\mathrm{E}}$ or $47.7 \mathrm{R}_{\mathrm{M}}$, as shown in Figure 2. This pass, like those shown in Figure 4, is typical of a number of passes in which there are no significant changes in the field at either satellite and only a portion of the +-+-+ pattern js identifiable.

A more interesting and less typical pass is shown in Figure 5b. The data were taken on September 13, 1967 about 3 hours after the beginning of a geomagnetic storm on earth, the SC occurring 
at $0345 \mathrm{UT}$. At $0700 \mathrm{UT}$ the two spacecraft were $16.0 \mathrm{R}_{\mathrm{E}}$ apart. Abrupt discontinuities are evident in the magnetic field at both spacecraft and the coherence between the two is remarkable. These events have been studied in more detail by Ness (1968) using individual data points at 5 second intervals. Inspection of even the 81.8 second sequence averaged data of Figure 5b shows that changes in the interplanetary magnetic field are found essentially undistorted in the lunar wake. This indicates that the effective response time of the moon is small compared with the time for the solar wind to convect the discontinuity past the moon.

The observations thus far presented have all been from regions of the lunar wake within about $3.5 R_{M}$ of the center of the moon. During the month of November the aposelene of the orbit of Explorer 35 swept through the lunar wake and thus during this period Explorer 35 made measurements of the wake at the greatest selenocentric radial distances. Figure 6 shows the orbits of Explorer 33 and the moon for the 1unar month from which data are presented in Figure 7. Figure $7 \mathrm{a}$ is a pass on November 25, 1967 while Figure $7 \mathrm{~b}$ is for November 28. During this period the spacecraft were separated by distances of 65 to $75 \mathrm{R}_{\mathrm{E}}$. In each of these passes the spacecraft did not enter the optical shadow as can be seen from the diagram in the upper right hand corner. Thus on these passes the spacecraft did not traverse the umbral region of the wake. This is perhaps the reason for 
the depression of the field measured by Explorer 35 during the November 25 pass. The satellite apparently spent its time in the penumbral decrease region and not in the umbral increase region. In neither of these passes is there a sharp increase in the field magnitude which might be identified as a shock nor any significant increase in the RMS deviations. Throughout the two passes, the RMS deviations are $0 F \leq 0.7 \gamma$ and $0.2 \gamma$, respectively, indicating a relatively steady field.

A similar pass taken on December 4, 1967 is shown in Figure 8 when the two spacecraft were $27.0 R_{E}$ apart. Although Explorer 35 did experience a short optical shadow on this pass it apparently did not enter the umbral region of the plasma shadow since the field magnitude is again somewhat depressed throughout the region around the shadow. Again there is no evidence of any tail shock in the data and the broad field magnitude decrease is evident. SUMMARY

The analysis of a number of passes of Explorer 35 through the lunar wake throughout the first 6 months of the spacecraft lifetime has substantiated the findings of earlier papers. In particular, there exists a characteristic pattern of perturbations of the interplanetary magnetic field in the lunar wake region. The perturbations are identifiable in the measurements of the magnitude of the field and consist of a series of increases and decreases relative to the magnitude of the undisturbed interplanetary magnetic field. 
Besides this +-+++ perturbation of the field magnitude, simultaneous two-satellite measurements of the vector magnetic field show that the presence of the moon does not significantly alter the interplanetary magnetic field. Changes observed in the undisturbed solar wind are found essentially undistorted in the lunar wake, and thus the response time of the moon to such changes is comparable to or faster than the time for convection of the change past the moon.

A search for a sudden increase in the field magnitude or fluctuations expected across a shock yielded negative results. The spacecraft mapped a region of the lunar wake out to about $5.4 R_{M}$ selenocentric radial distance and as yet nothing has been found that resembles the tail shock predicted by Michel (1967). However, at the large radial distances down the wake Explorer 35 was approximately $1 R_{\mathbb{M}}$ from the center of the optical shadow.

Apart from the small wake perturbations mainly evident in the field magnitude, the presence of the moon has no other identifiable effect on the interplanetary magnetic field. These results do not support the earlier report of a lunar wake at $150 \mathrm{R}_{\mathrm{M}}$ downstream of the moon based on data obtained by IMP I (Ness, 1965). The magnetic effects of the moon on the interplanetary magnetic field can be summarized by the statement that the moon behaves like a poorly conducting, nonmagnetic body with a negligible permanent magnetic field. 


\section{ACKNOWLEDGEMENTS}

We are indebted to Dr. Y. C.Whang for many helpful discussions about this problem. We also wish to thank C. S. Scearce and Dr。S。C. Cantarano for their help with the engineering aspects of the experiment. 


\section{REFERENCES}

Behannon, Kenneth W., "Mapping of the Earth's Bow Shock and Magnetic Tail by Explorer 33", J. Geophys. Res., 73, 907-930, 1968.

Colburn, D. S., R. G. Currie, J. D. Mihalov and C. P. Sonett, "Diamagnetic Solar Wind Cavity Discovered Behind Moon," Science, $158,1040-1042,1967$.

Lyon, E. F., H. S. Bridge and J.H. Binsack, "Explorer 35 Plasma Measurements in the Vicinity of the Moon," J. Geophys. Res., $72,6113-6117,1967$.

Michel, F. C., "Magnetic Field Structure Behind the Moon," J. Geophys. Res., 73, 1533-1542, 1968 .

Ness, N. F., "The Magnetohydrodynamic Wake of the Moon," J. Geophys. Res. , 65, 517-534, 1965 .

Ness, N. F., "Electrical Conductivity of the Moon," Trans. Am. Geophys. Union, 49,242 , 1968. Abstract.

Ness, N. F., K. W. Behannon, C. S. Scearce and S. C. Cantarano, "Early Results from the Magnetic Field Experiment on Lunar Explorer 35," J. Geophys. Res. , 72, 5769-5778, 1967.

Ness, N. F., K. W. Behannon, H. E. Taylor and Y. C. Whang, "Perturbations of the Interplanetary Magnetic Field by the Lunar Wake," J. Geophys. Res., 73, June 1, 1968.

Spreiter, J. R., M. C. Marsh, A. L. Summers and A. Y. Alksne, "Interactions of the Solar Wind with the Moon: Theoretical Considerations," Trans. Am. Geophys. Union, 49, 234, 1968. Abstract.

Whang, Y. C., "Theoretical Study of the Magnetic Field in the Lunar Wake," GSFC Research Report X-612-68-17, January 1968. To appear in September 1968 Phys. of Fluids. 
Figure 1. Selected orbits from the first six months after launch of Explorer 35, projected into the selenocentric solar ecliptic $x, y$ plane. Distances are in lunar radii. Each orbit is labeled near aposelene with its date.

Figure 2. An $\mathrm{x}, \mathrm{y}$ geocentric solar ecliptic projection of the orbits of Explorers 33, 34 and 35. Distances are in earth radii. Simultaneous Explorer 33, 35 data are presented from the period September 9-13 when both spacecraft were near the dusk meridian.

Figure 3. Simultaneous Explorer 33 - Explorer 35 magnetic field measurements presented in solar ecliptic coordinates. $F$ is the field magnitude in gammas, $\theta$ its latitude angle $\left(0^{\circ}\right.$ is in the ecliptic plane and positive angles are northward), and $\varphi$ is the azimuth angle $\left(0^{\circ}\right.$ is toward the sun and $90^{\circ}$ toward dusk.) $\delta F$ is the standard deviation of the 16 measurements of the field magnitude in each data point. The position of Explorer 35 relative to the moon is shown in ecliptic plane projection in the upper left for each pass. The diagram in the upper right of each pass is a projection of the orbit on a plane perpendicular to the moon-sun line. The $\mathrm{Z}$ axis in this diagram taken to be perpendicular to the 
average magnetic field, in the direction of $\langle\overrightarrow{\mathrm{B}}>\mathrm{x} \overrightarrow{\mathrm{X}}$. The optical shadow on Explorer 35 is indicated. The + and - signs indicate regions where the field magnitude measured by Explorer 35 is respectively greater than and less than that at Explorer 33. The value of the 3-hour terrestrial magnetic activity index $K_{p}$ at the time of the pass is also given.

Figure 4. Same format as Figure 3. The measurements by both spacecraft indicate that the interplanetary field was relatively steady during this period of low $\mathrm{K}_{\mathrm{p}}$.

Figure 5. Same format as Figure traversal of the

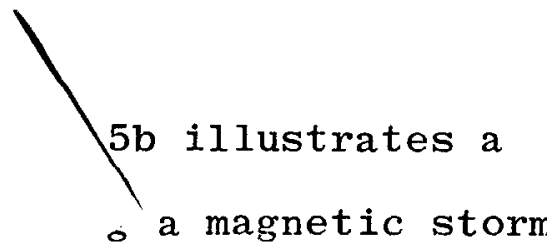
on earth.

Figure 6. Orbi and the moon for the fifth - launch of Explorer 35 as in Figure seous data are presented for 3 passes , the period shown here: November 25, November .6 and December 4.

. Same format as Figure 3. Note that Explorer 35 does not enter the optical shadow on these passes.

Figure 8. Same format as Figure 3. Explorer 35 is seen to be shadowed by the moon only briefly during this pass. 


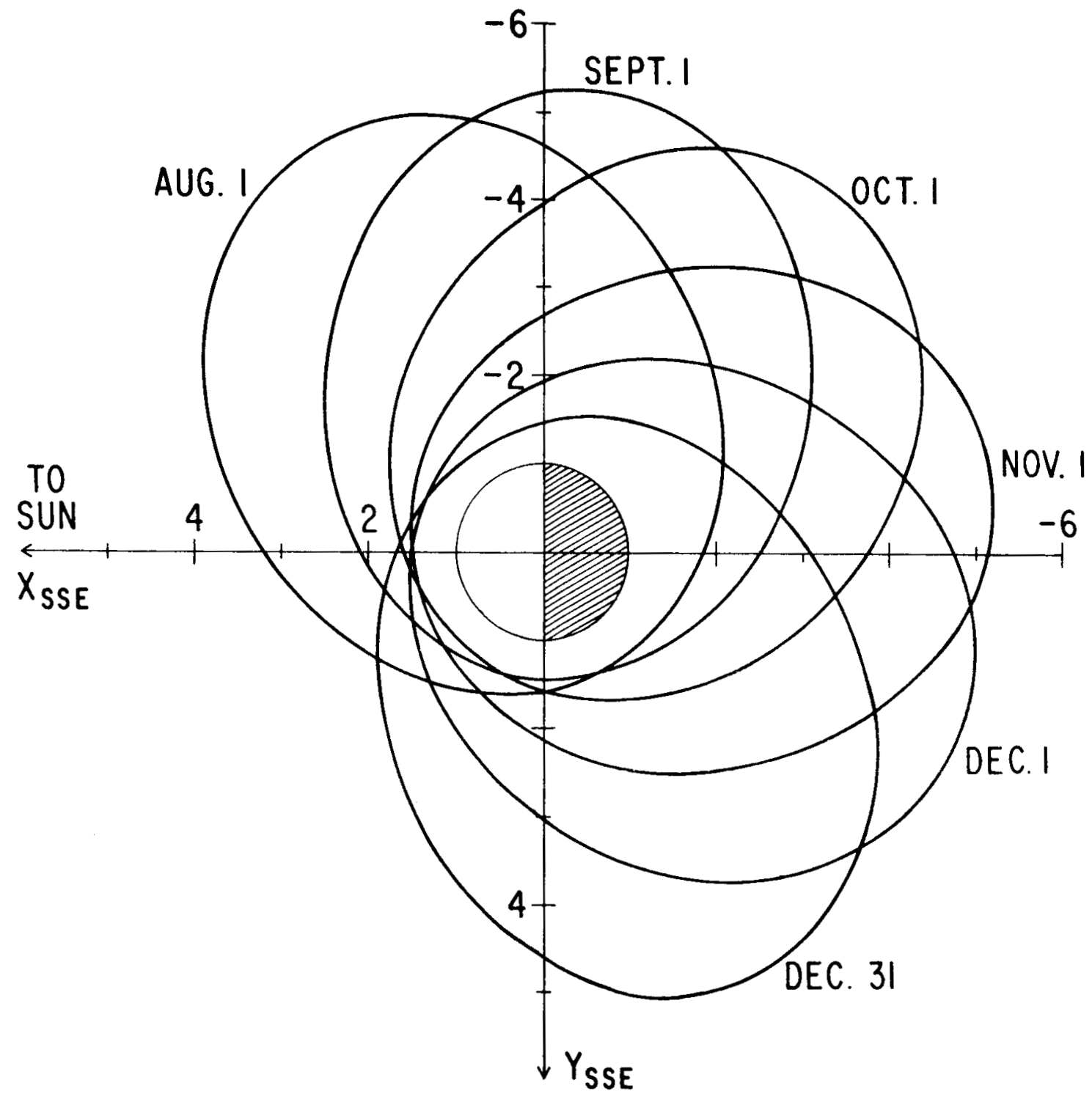

EXPLORER 35 ORBITS, 1967

Figure 1 


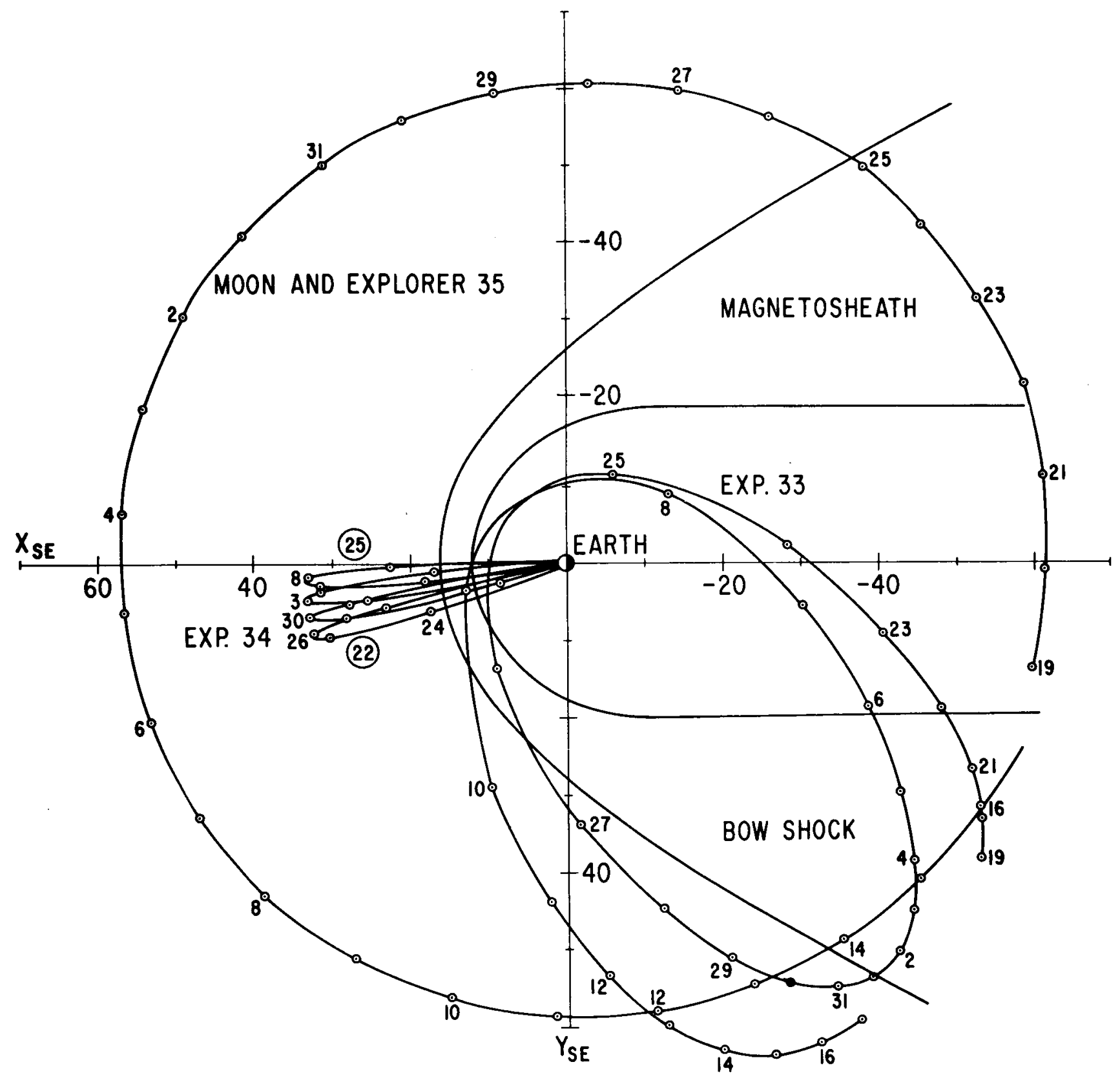

AUGUST-SEPTEMBER 1967

Figure 2 


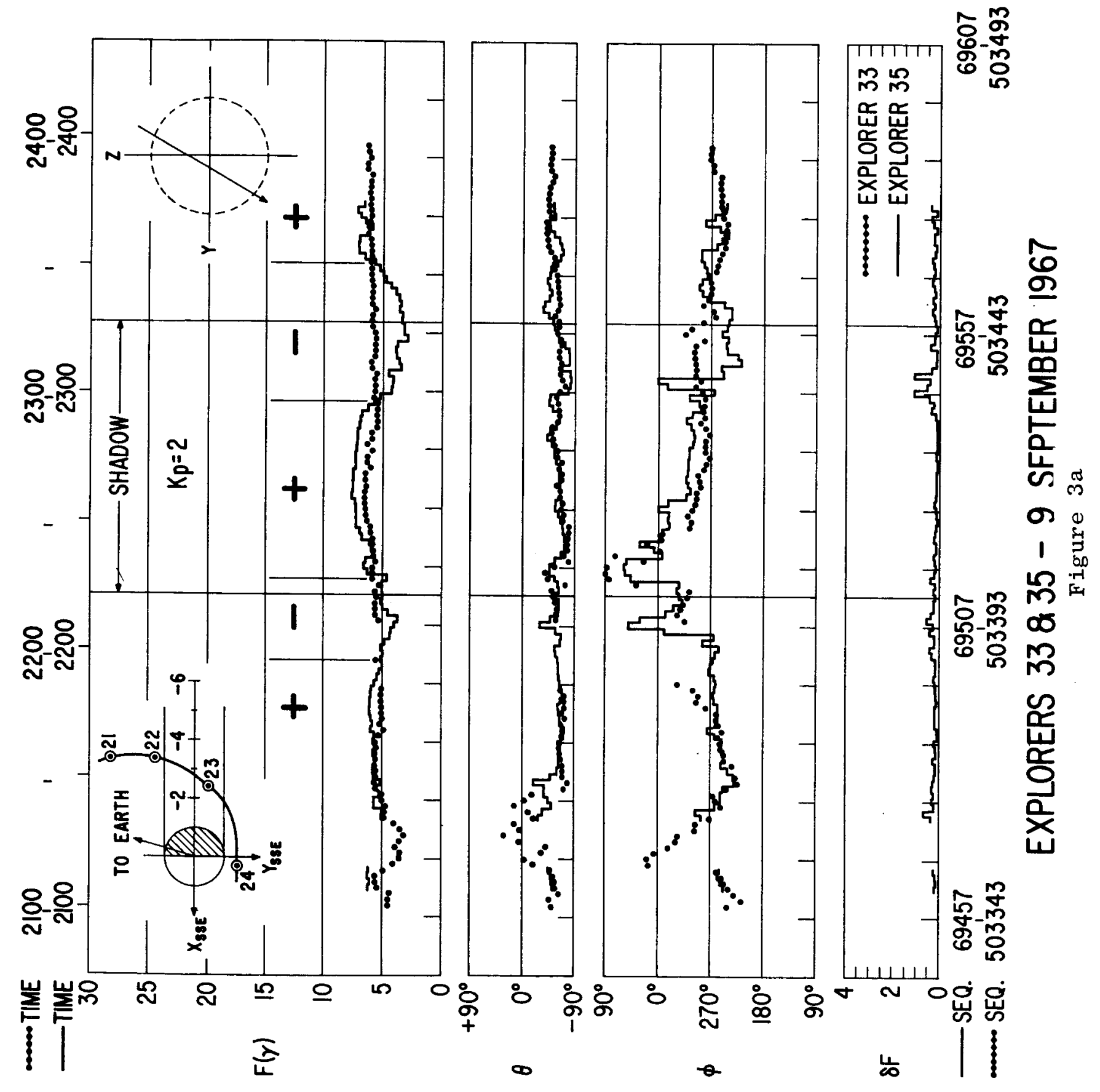




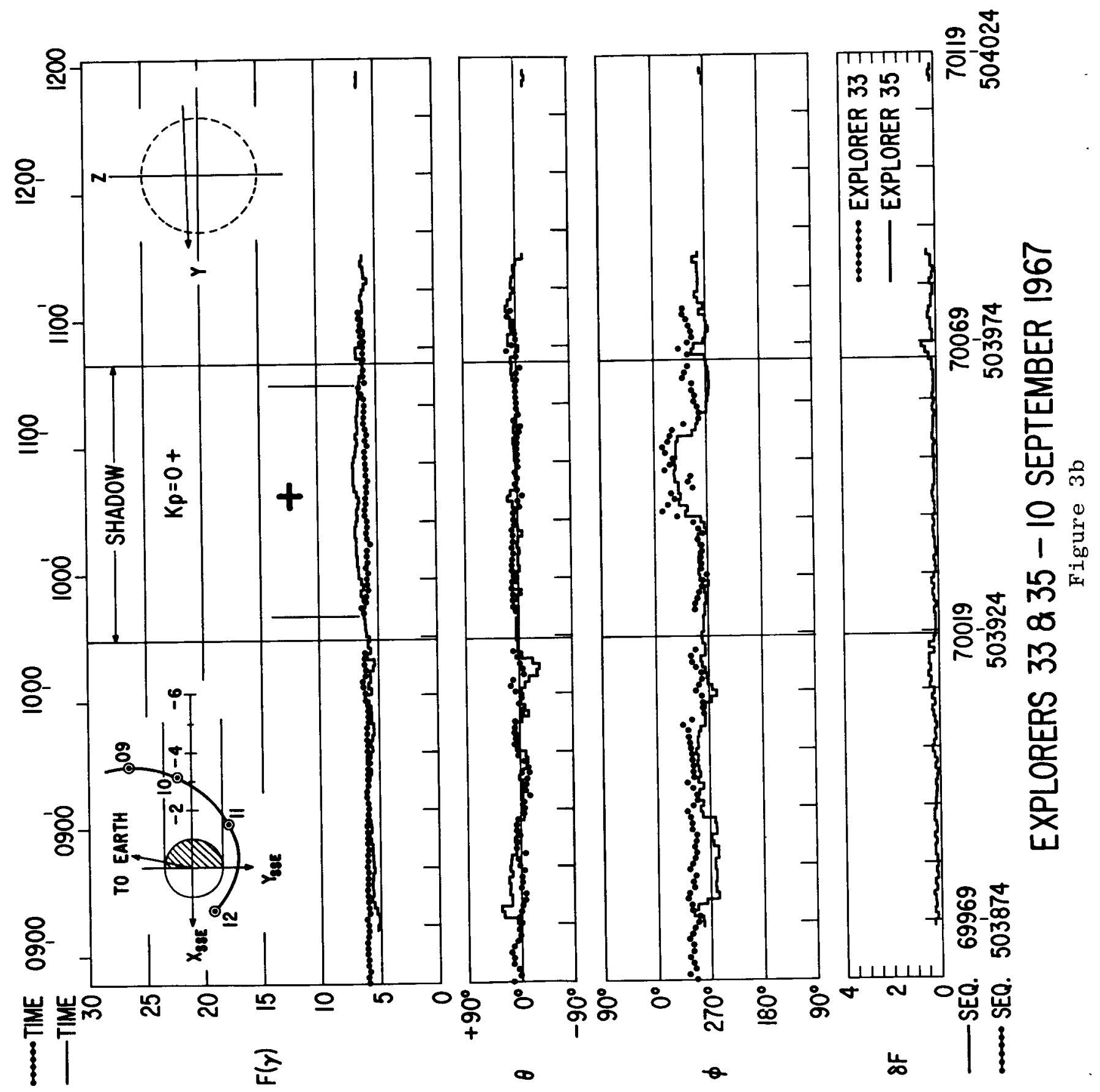




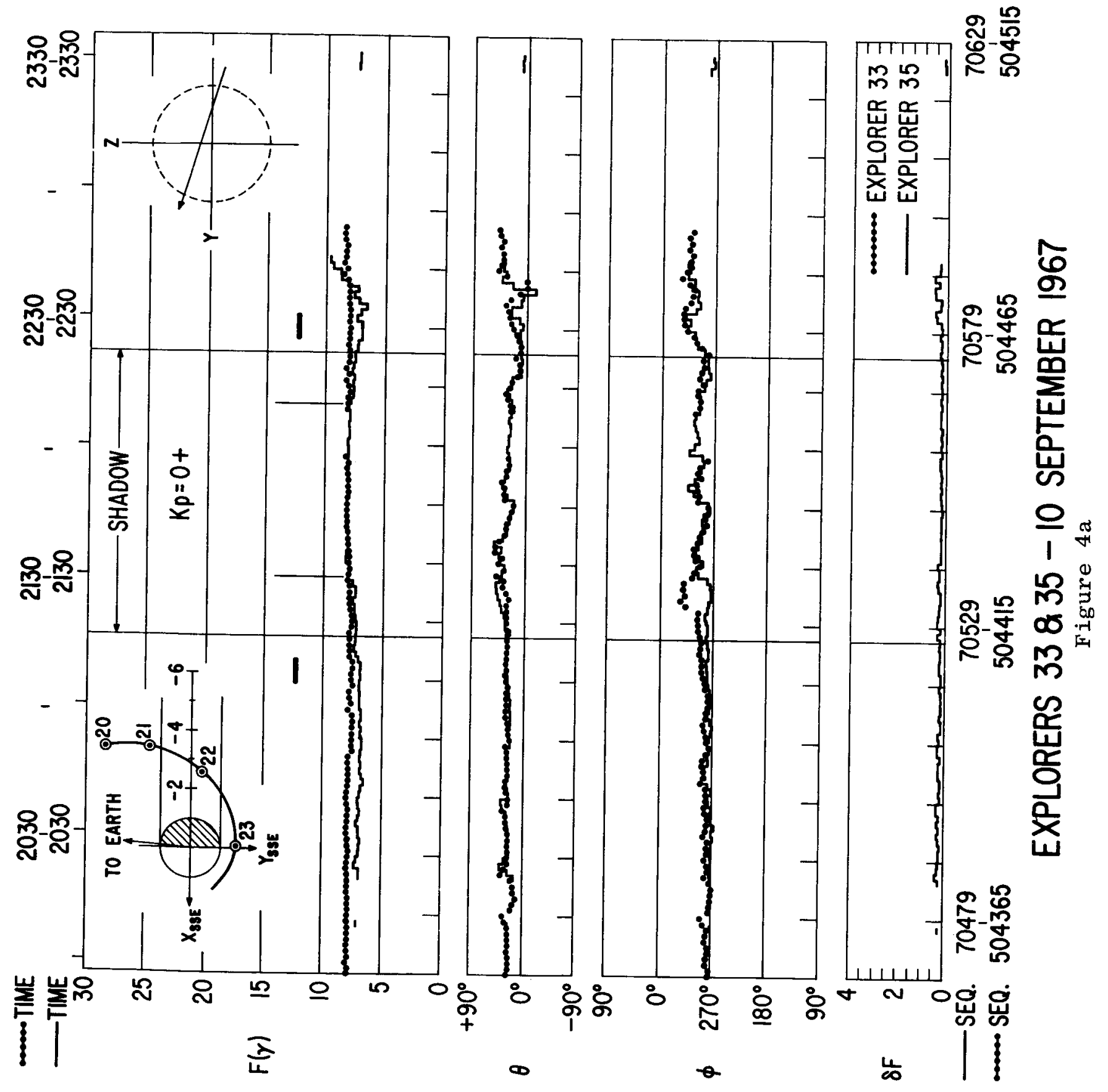




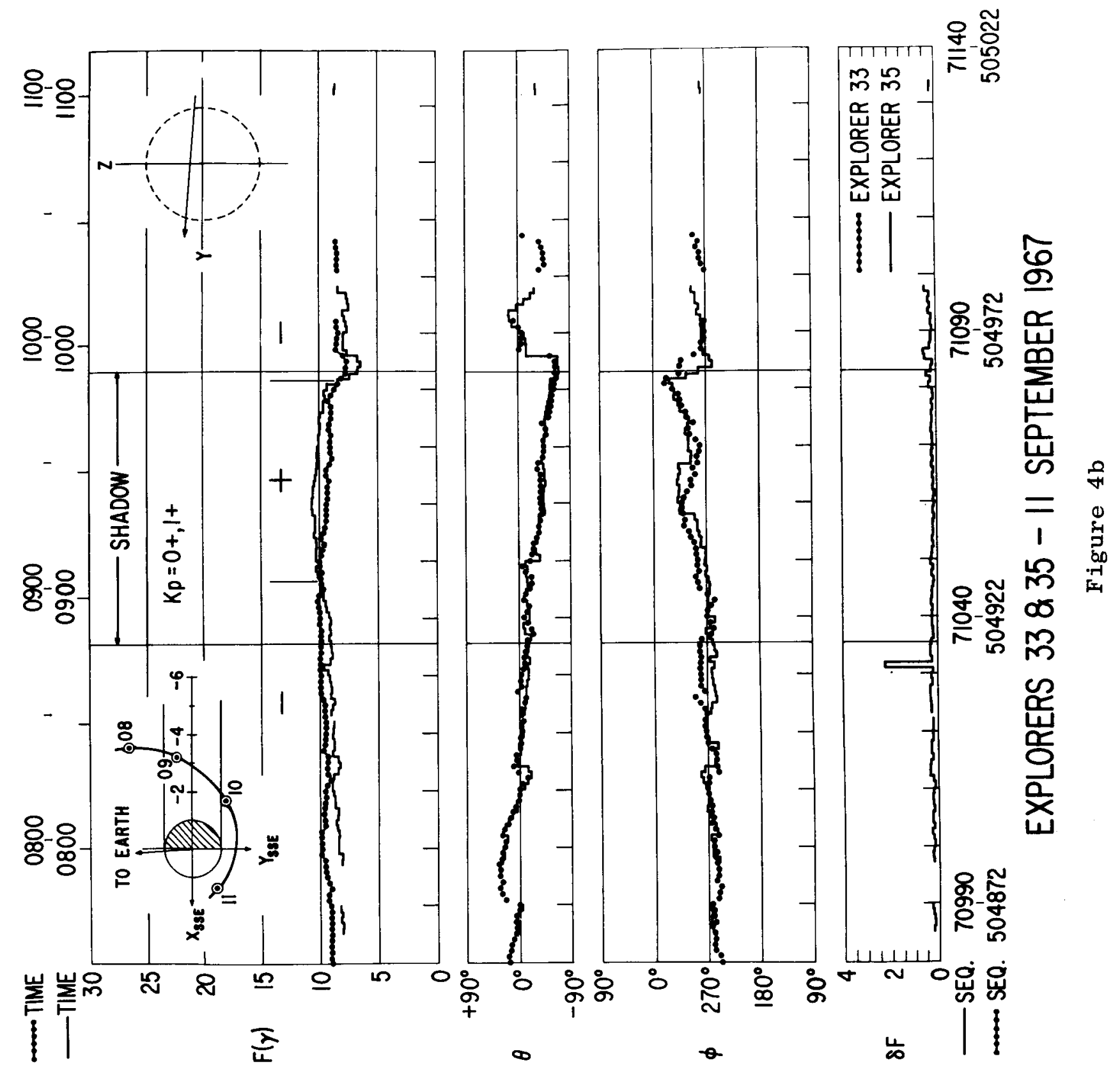




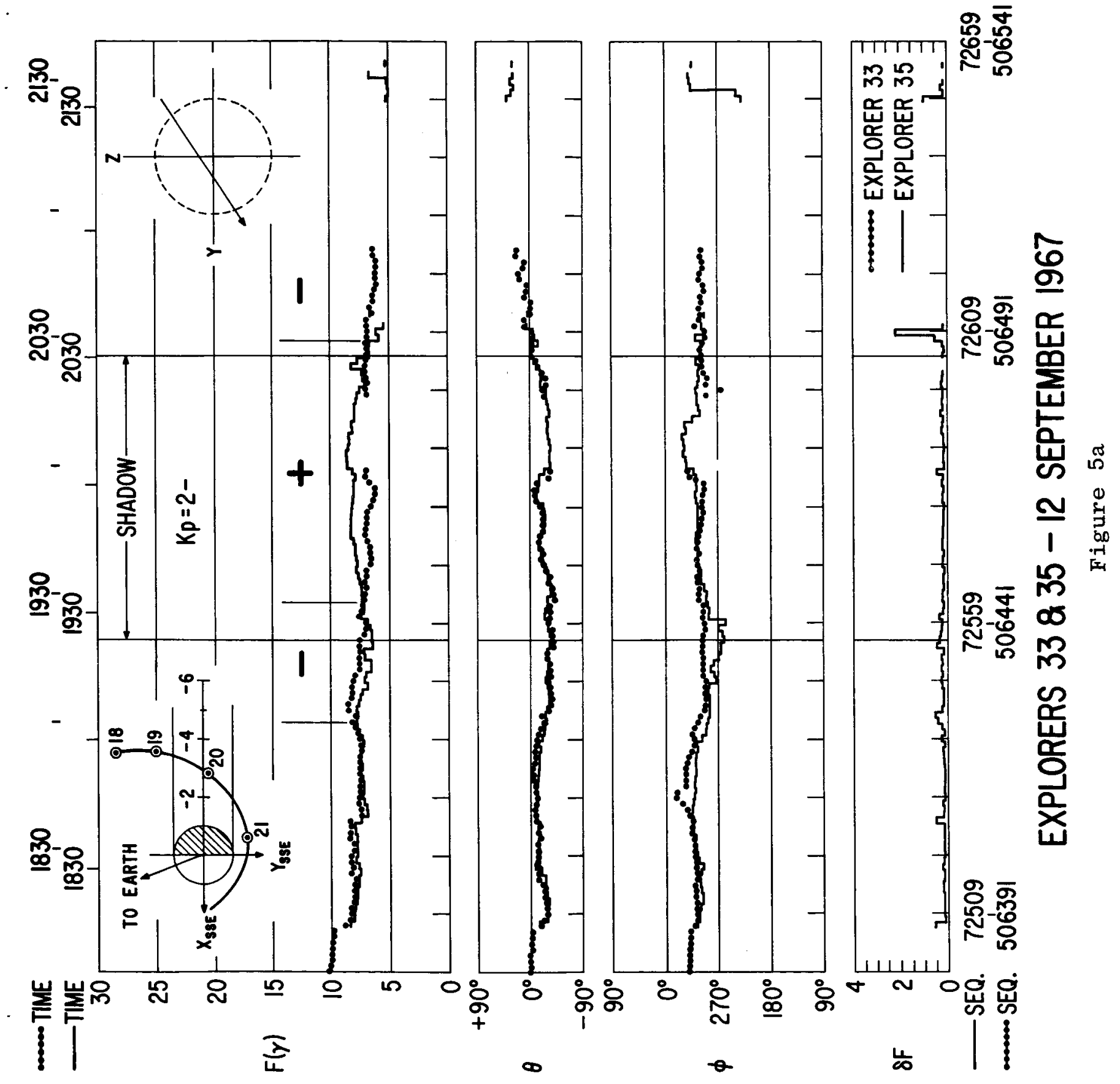




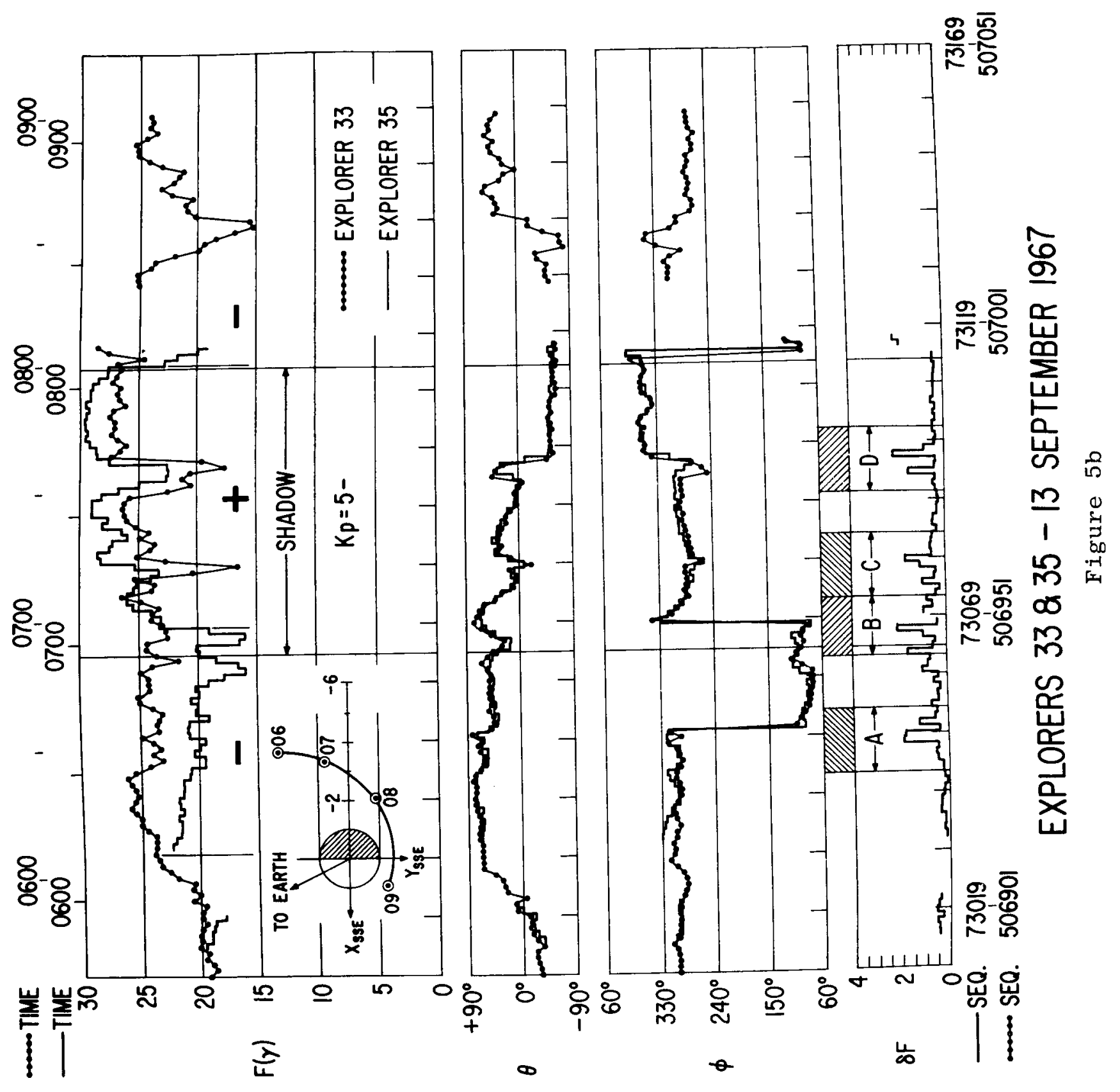




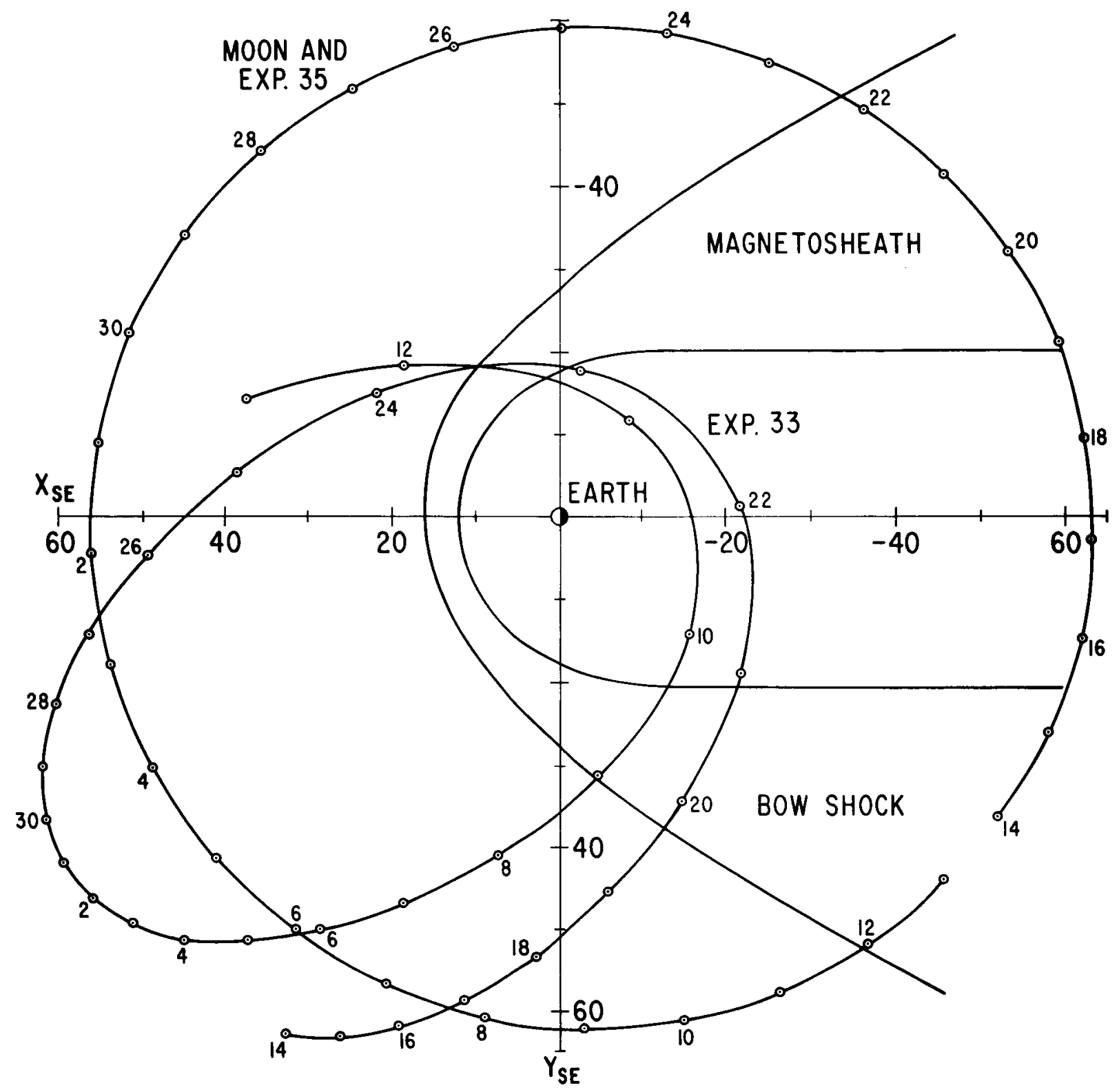

NOVEMBER-DECEMBER 1967

Figure 6 


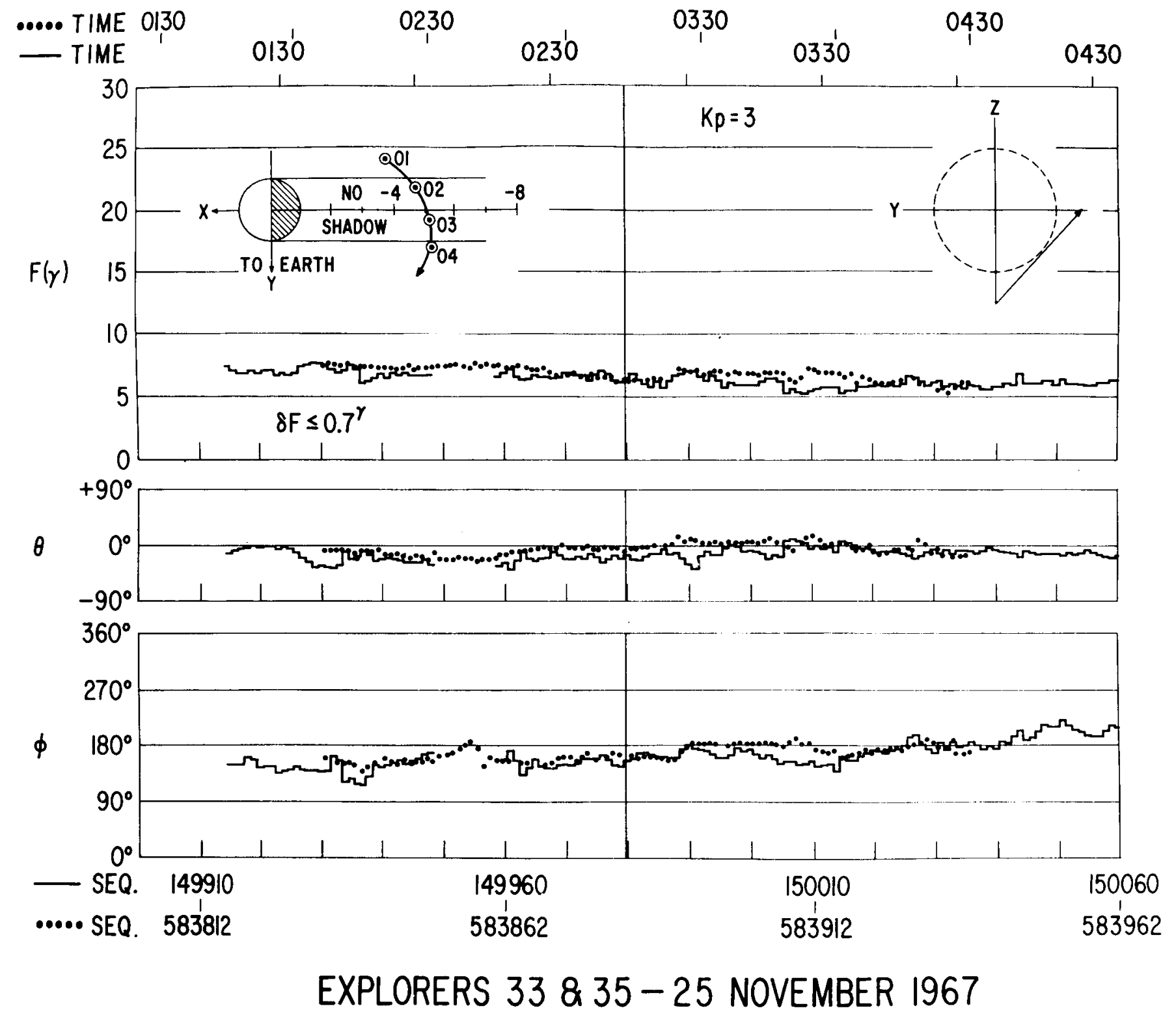

Figure $7 a$ 

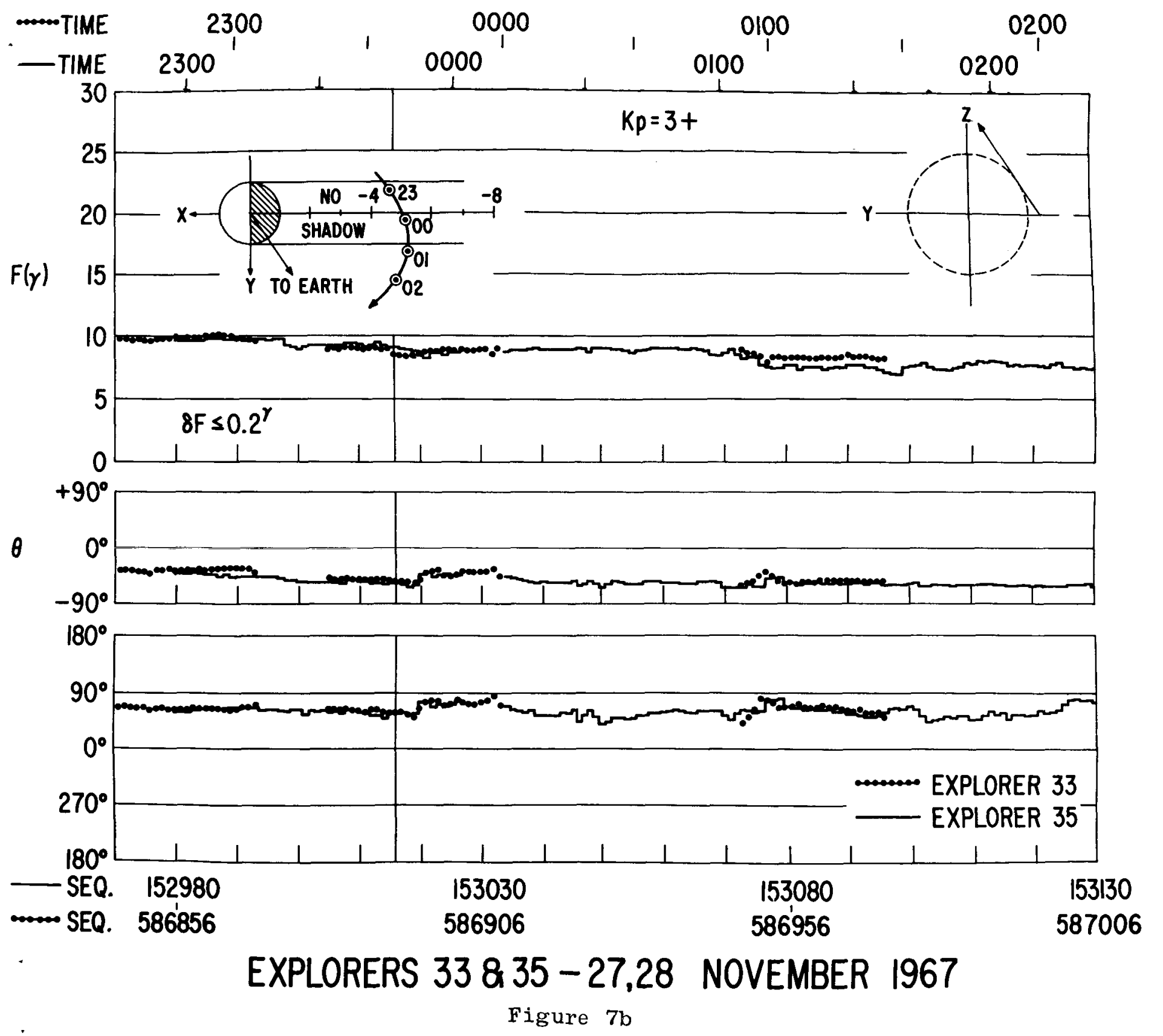

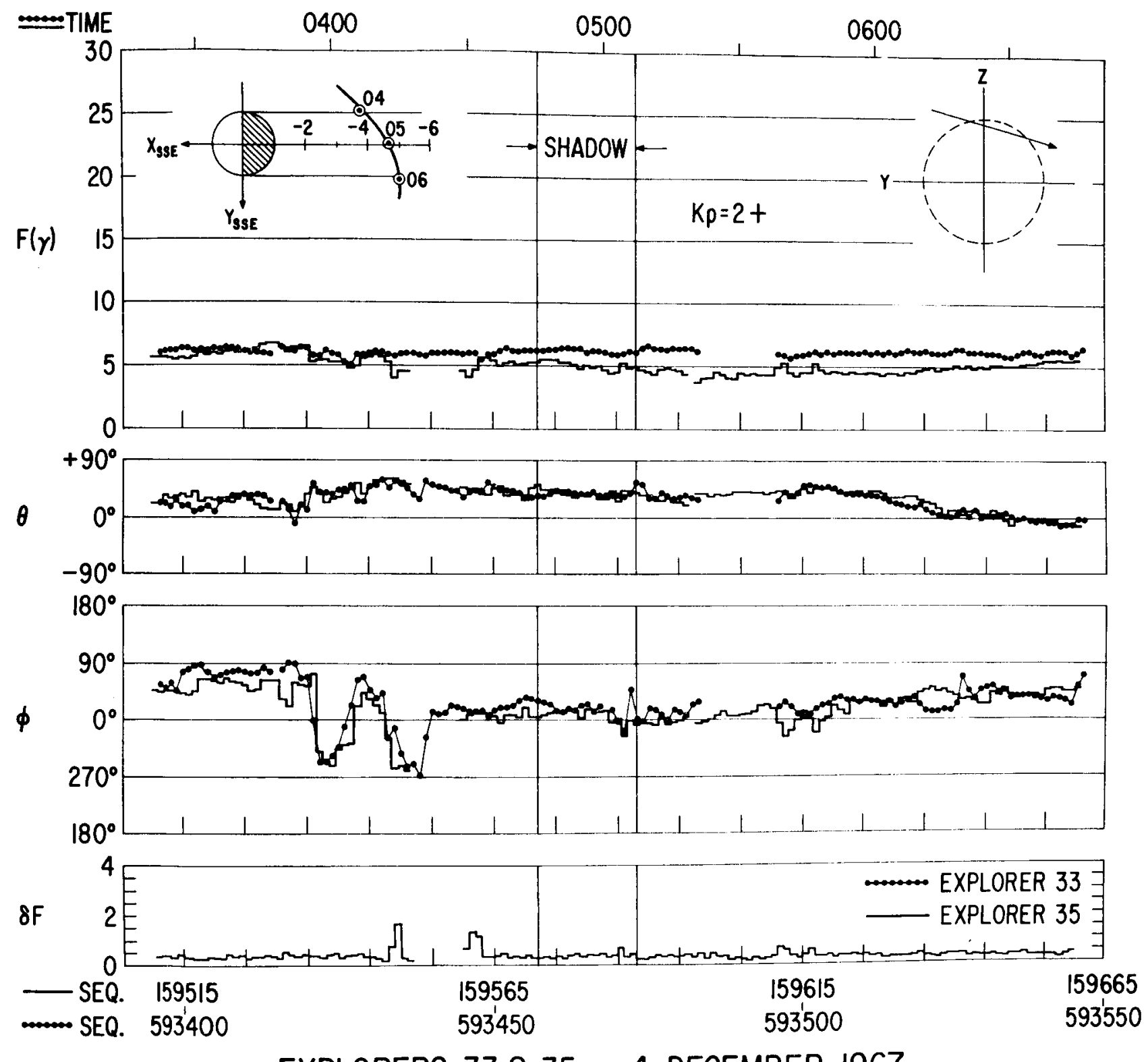

EXPLORERS $33 \& 35$ - 4 DECEMBER 1967

Figure 8 